\begin{tabular}{|c|c|c|c|c|c|c|c|c|c|}
\hline $\mathrm{Nr}$. & A III4 & A I $38 \mathbf{I}$ & A I 386 & $\begin{array}{r}\mathrm{Pla} \\
\mathrm{A} I 73^{8} \\
\end{array}$ & B 84 & A 2000 & B 118 & B 378 & Bemerkungen \\
\hline 13 & $13 \frac{\mathrm{m}}{.8}$ & $<\mathrm{I} 4^{\mathrm{in} !}$ & $14 \%$ & $14 \stackrel{m}{\circ}$ & $12 \div 5$ & $13^{m} \cdot 5$ & $13^{m} \cdot 3$ & I $2 \stackrel{m}{m} \cdot 3$ & \multirow{12}{*}{$\begin{array}{l}\text { auf } 84 \text { wohl um ca. } 1^{\mathrm{m}} \text { heller als auf } \text { i } 18 \\
\text { wohl } 2 \text { weifellos kurzperiodisch }\end{array}$} \\
\hline 14 & 13.2 & 12.0 & 11.8 & 12.5 & $12 \cdot 3$ & 12.0 & $12 \cdot 3$ & 12.7 & \\
\hline 15 & $?$ & - & - & - & - & - & - & $?$ & \\
\hline I 6 & $<$ I $_{4}$ & 12.8 & $<\mathbf{1 4 . 2}$ & $<14$ & 13.5 & 13.5 & 13.0 & $<15.0$ & \\
\hline 17 & 13.8 & $<14$ & 13.8 & 12.5 & 15.0 & I 3.5 & 15.0 & 13.8 & \\
\hline 18 & 13.2 & $13 \cdot 3$ & 13.0 & $<14$ & I 3.8 & $<14$ & 12.8 & 12.6 & \\
\hline 19 & 11.8 & 11.8 & 13.2 & 11.8 & $<14.5$ & $<14$ & 12.3 & 11.8 & \\
\hline 20 & $<14$ & 14.0 & $<14.2$ & 14.0 & 13.5 & $<14$ & 13.5 & 15.0 & \\
\hline 2 I & 12.8 & 11.8 & 12.5 & 13.0 & 12.5 & 12.5 & 12.5 & 11.7 & \\
\hline 22 & 14.0 & $<\mathbf{I}_{4}$ & $<14$ & 13.0 & 13.9 & $<14$ & 13.4 & 15.0 & \\
\hline 23 & I I. 5 & 10.0 & 9.8 & $<14$ & $<15.2$ & $<\mathrm{I}_{4}$ & $<15.2$ & 10.0 & \\
\hline 24 & 13.2 & 13.5 & 13.8 & I 3.8 & 14.5 & 14.0 & 14.5 & 14.5 & \\
\hline 25 & I 3.0 & 12.8 & 12.8 & 13.0 & 12.4 & I 2.4 & I 2.4 & 12.4 & \multirow{11}{*}{$\begin{array}{l}\text { scheint im ganzen heller geworden } \\
\text { wohl sicher auch variabel }\end{array}$} \\
\hline 26 & 12.8 & 13.5 & 14.2 & 13.2 & 12.8 & $<14$ & 12.8 & 13.2 & \\
\hline 27 & 13.0 & 14.0 & 13.8 & $13 \cdot 3$ & 13.0 & 13.2 & 12.8 & 12.8 & \\
\hline 28 & 13.0 & I 2.8 & 12.5 & 13.0 & 13.0 & $<I_{4}$ & 13.0 & 12.8 & \\
\hline 29 & 13.0 & 13.8 & 13.2 & 13.0 & 13.0 & 135 & 13.0 & 13.0 & \\
\hline 30 & 13.0 & 14.0 & 13.5 & 13.5 & 13.0 & 13.2 & 13.0 & 13.2 & \\
\hline $3 \mathrm{I}$ & 13.2 & 12.8 & 12.9 & 12.6 & 12.5 & 13.2 & 12.5 & 12.7 & \\
\hline 32 & $S>N$ & $\mathbf{N}>\mathbf{S}$ & $\mathbf{N}>\mathbf{S}$ & $\mathbf{N}>\mathbf{S}$ & $\mathrm{S}>\mathrm{N}$ & $\mathrm{N}>\mathrm{S}$ & $\mathbf{N}>\mathbf{S}$ & $\mathrm{S}>\mathrm{N}$ & \\
\hline 33 & 14.0 & 14.0 & 14.0 & 14.0 & 13.2 & 13.8 & 13.2 & 13.5 & \\
\hline 34 & 13.2 & 13.4 & 13.4 & 13.5 & $13 \cdot 3$ & I 3.9 & 12.8 & 13.4 & \\
\hline 35 & I $3 \cdot 5$ & 13.0 & 13.5 & 13.5 & 12.7 & 13.0 & 12.7 & 12.7 & \\
\hline
\end{tabular}

Wie ersichtlich sind einzelne der Variabeln ganz interessante Objekte, welche sehr starke Veränderlichkeit zeigen. Ich hebe außer Nr. I und Nr. 15 besonders die Variabeln:

$3,6,7,8,10,11,16,17,18,19,20,22$ und 23

hervor, die leicht und sicher als veränderlich nachzuweisen sind; darunter sind wieder die Nummern 3, 7, I $1,16,17$ und ganz besonders Nr. 23 beachtenswert, der beinahe um 6 Größenklassen in seinem Lichte schwankt.

Es kam mir hier selbstverständlich zuerst darauf an, zu zeigen, was der Stereokomparator bei den Veränderlichen zu leisten im Stande ist, und ich hoffe mit vorstehendem meinem Versprechen von A.N. 3749 nachgekommen zu sein.
Den Orionnebel habe ich deshalb dazu gewählt, weil sich aus den Schlüssen, die sich uber den Zusammenhang des Orionnebels mit den Sternleeren ergaben, vermuten ließ, daß hier wohl etwas derartiges gefunden werden könnte. In der Tat ist der hiermit nachgewiesene Reichtum des Orionnebels an verănderlichen Sternen sehr auffallend. Die meisten aufgefundenen Variablen - die oben besonders hervorge. hobenen ohne Ausnahme - befinden sich in mehr oder minder dichten Nebelpartien dieser Gegend und stehen wohl mit der Entwickelung dieses gewaltigen Nebelgebildes in engstem Zusammenhang, wie es ahnlich schon $O$. Struve von anderen Gesichtspunkten ausgehend vermutet hat.

\title{
Bemerkungen und Berichtigungen zur Bonner Durchmusterung.
}

In den Monthly Notices Vol. LXIII p. 420 teilt Herr! die Stelle 1856 Mărz 25 am Meridiankreise und fand, wie Burnham mit, dab er die Sterne

$$
\begin{array}{llll}
\mathrm{BD} .+10.2117 & 9^{\mathrm{m}} \cdot 5 & 10^{\mathrm{h}} 3^{\mathrm{m}} 17^{\mathrm{s}} .2 & +10^{\circ} 37: 3 \text { und } \\
\mathrm{BD} .+10.2118 & 9.5 & 103^{25.2}+1038.3
\end{array}
$$

am Himmel vermißt habe. Ich habe deshalb die Originale der BD. nachgesehen und folgendes gefunden.

In den Sucherzonen ist an dieser Stelle nur einmal ein Stern beobachtet, nămlich in S. Z. 104 Sch. 1853 März ro, Luft mảBig gut,

$$
9 \% \quad 10^{\mathrm{h}} 3^{\mathrm{m}} 18^{\mathrm{s}} \cdot 3+10^{\circ} 38: 3 .
$$

Da dieser Stern neunter Größe in der zweiten Sucherzone 403 , die über die Gegend ging, fehlte, so revidierte Argelander dies so hăufig vorkommt, da $\mathrm{z}$ wei schwache Sterne dort nahe beieinander standen, die er durch Austritt aus dem dunklen Feld wie folgt beobachtete:

$$
\begin{array}{lll}
9^{\mathrm{m}} \cdot 5 & 10^{\mathrm{h}} 3^{\mathrm{m}} 17^{\mathrm{s}} \cdot 2 & +10^{\circ} 37: 3 \\
9.5 & 103^{25} \cdot 2 & +1038.3
\end{array}
$$

Der voraufgehende ist in Dekl. nur geschätzt gegen den folgenden und sein Ort deshalb später in den Katalog der Meridianörter in BB. VI nicht aufgenommen. Dieser Meridianbeobachtung entsprechend hat Argelander beide Sterne in die BD. eingereiht, wobei er offenbar mit Recht annahm, $\mathrm{da} B$ in der Sucherzone $10_{4}$ ibr vereintes Licht zu der ubertriebenen Schätzung 9.0 Veranlassung gegeben hatte. Beide 
Sterne wurden außerdem bestätigt durch Hind's ecliptical chart, wo sie als I $^{\text {m }}$ verzeichnet sind; ferner ist der voraufgehende auch im Markree Catalogue beobachtet:

$$
10^{\mathrm{m}} 10^{\mathrm{h}} 3^{\mathrm{m}} 19^{\mathrm{s}}+10^{\circ} 3^{6: 7} \text {. }
$$

Nach diesem Befunde erschien mir die Existenz beider Sterne gesichert, doch war es wahrscheinlich, daß sie zu den schwachen der Klasse 9.5 gehören würden, da sie im Meridiankreise nur im dunklen Feld beobachtet waren und bei Hind als $1^{\mathrm{m}}$ vorkamen. Eine Beobachtung am Bonner Re. fraktor 1903 Juni 15, allerdings tief am Horizont in Dämmerung und Dunst, hat dies bestätigt. Ich fand beide Sterne als $10^{\mathrm{m}} 3$ an ihrem $\mathrm{BD}$. Ort und maß fluchtig folgende Diffe. renzen gegen + 10.2119 , dessen $B D$. Ort genau ubereinstimmt mit dem scharfen Ort in Bonper Veröff. Nr. 4 p. 64 und dessen Größe ich dort zu 9.3 geschätzt habe:

$$
\begin{array}{rrrr} 
& \multicolumn{2}{c}{\text { in BD. }} \\
-16.5 & -6: 5 & -17^{5} .1 & -6: 0 \\
-9.2 & -5.2 & -9.1 & -5.0
\end{array}
$$

also durchaus ubereinstimmend mit der BD., die somit keiner Verbesserung bedarf. Den von Burnham gemessenen nahen Begleiter von +10.2IIg konnte ich nicht sehen; er muß, uuter den angeführten Umständen, also wohl schwächer als $11^{\mathrm{m}}$ sein, falls er wirklich bei $+10: 21 \mathrm{~s} g$ steht.

Durch Herrn Dr. Ristenpart bin ich auf folgende gröbere Fehler der BD., die in der 2. Auflage noch nicht berichtigt sind, aufmerksam geworden.

$+1: 2129$ statt 19:4 lies 15:9, unter der plausiblen Annahme, daß die eine Sucherbeobachtung um - I Teil zu korrigieren sei; es wird dadurch Übereinstimmung mit dem AG. K. Albany hergestellt, wo die Örter von 3433 und 3434 beide $z u+1: 2128$ gehören, während 3435 sich auf $+1: 2129$ bezieht. - Die Karte ist in beiden Auflagen entsprechend zu verbessern.

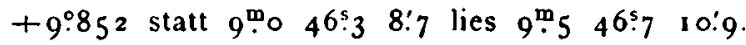

$+9: 856$ statt 9.3 lies $8: 9$.

Diese Stelle ist bereits im AG. K. Leipzig II p. 247 besprochen worden; eine nochmalige genaue Durchsicht der Originale läßt mir keinen Zweifel, daß sie, wie eben angegeben ist, zu korrigieren sei. Die Karte ist in beiden Auflagen gleichfalls 20 berichtigen.

$+10^{\circ} .40288^{\mathrm{m}} 2^{\mathrm{h}} 52^{\mathrm{m}} 5^{6: 8}+10^{\circ} 16.5$ statt $52^{\mathrm{m}}$ lies $53^{\mathrm{m}}$; die Karte ist in beiden Auflagen schon richtig.

$+10: 2494$ statt $56: 7$ lies $47: 4$; ein Schreibfehler im Hauptkatalog. Beide Karten sind schon richtig.

$+21^{\circ} \cdot 32029^{\mathrm{m}} \cdot 517^{\mathrm{h}} 3^{8^{\mathrm{m}}} 3^{\mathrm{s}} \mathrm{s}+21^{\circ} 33^{\prime} \cdot 2$ ist im Druck in beiden Auflagen richtig, dagegen haben beide Karten den Stern falsch bei $+2 I^{\circ} 53^{\prime} \cdot 2$ eingezeichnet.

$+53^{\circ} 3099$ ist identisch mit $+52^{\circ} .3395$ und in $53^{\circ}$ zu streichen, wie schon BB.V p. XXXV angegeben war. Die Karten haben richtig nur einen Stern.

$+61910089^{\mathrm{m}} \cdot 47^{\mathrm{h}} 42^{\mathrm{m}} 59^{\mathrm{g}}+61^{\circ} 4: 3$ statt $42^{\mathrm{m}}$ lies $41^{\mathrm{m}}$ auch die Karte ist in beiden Auflagen zu berichtigen.

Endlich können folgende, einmal in den BD. Originalen vorkommende Sterne nachgetragen werden:

$$
\begin{array}{rrrrr}
+1.4513 \mathrm{a} & 9^{\mathrm{m}} \cdot 5 & 21^{\mathrm{h}} 30^{\mathrm{m}} 41^{\mathrm{s}} \cdot 6 & +1011: 0 \\
+28.1280 \mathrm{a} & 9.3 & 64728.2 & +28 & 0.7 \\
+28.4536 \mathrm{a} & 9.5 & 23 & 238.8 & +2817.5
\end{array}
$$

letzterer wiederholt aus BB.VI p. 378 .

Bonn, I 903 Juli 6.

\section{F. Kilstner.}

\begin{tabular}{|c|c|c|c|c|c|c|c|}
\hline No. & Gr. & $\alpha$ & 190 & 0.0 & $\delta_{\mathrm{I}}$ & 900 & 0.0 \\
\hline 1 & $12.1-12.1$ & $o^{h}$ & & $20: 31$ & $+60^{\circ}$ & 2 & $15^{\circ}$ \\
\hline 2 & $12.0-12.0$ & 0 & 4 & 25.07 & +60 & 6 & $27 \cdot 32$ \\
\hline 3 & $11.0-1$ & 0 & 5 & $6.5 \mathrm{I}$ & $+6 I$ & 6 & 10 \\
\hline 4 & $8.0-10.0$ & 0 & 17 & 23.88 & +61 & 41 & 12.43 \\
\hline 5 & $11.0-11.0$ & 0 & 17 & 26.16 & +60 & 38 & 52.35 \\
\hline 6 & $10.0-10.0$ & 0 & 17 & 57.47 & +59 & 57 & 27.78 \\
\hline 7 & 11.0 & 0 & 18 & & +60 & 23 & I $3 \cdot 3^{8}$ \\
\hline 8 & $8.0-12.0$ & $\circ$ & 18 & 21.18 & +60 & 16 & 58.0 \\
\hline 9 & $11.0-11.0$ & $o$ & 19 & 47.19 & +62 & 3 & 5.88 \\
\hline 10 & $9.0-11.0$ & 0 & 21 & 33.69 & +60 & 39 & 11.83 \\
\hline I I & $12.0-11.0$ & $\circ$ & 22 & 35 & +61 & 9 & 36.81 \\
\hline 12 & $11.0-12.0$ & 0 & 26 & 14.30 & +60 & 31 & 14.29 \\
\hline 13 & $9.0-11.0$ & o & 26 & 16.24 & +59 & 34 & 36.64 \\
\hline 14 & $10.0-10.0$ & 0 & 26 & 29.07 & +60 & 4 & 34.41 \\
\hline 15 & $11.0-12.0$ & 0 & 26 & 29.37 & +60 & 5 & 27.99 \\
\hline 16 & $10.5-11.0$ & 0 & & 51.84 & +60 & 17 & 50.61 \\
\hline 17 & $10.9-12.0$ & $\circ$ & 28 & 55.34 & +59 & 42 & 37.23 \\
\hline
\end{tabular}

\section{Elenco delle stelle doppie}

\begin{tabular}{|c|c|c|c|c|c|c|}
\hline No. & Gr. & $\alpha 190$ & دo.० & & 1900 & 0.0 \\
\hline 18 & $12.0-12.0$ & $o^{h} 29^{m}$ & 6510 & $+60^{\circ}$ & $2^{\prime}$ & $41: 49$ \\
\hline 19 & $10.0-11.0$ & - 3 I & 8.87 & +59 & 57 & $5^{2} \cdot 3^{8}$ \\
\hline 20 & $11.0-12.0$ & o 33 & 27.34 & $+6 i$ & 34 & 17.02 \\
\hline 21 & $11.5-11.4$ & - 33 & 46.77 & +59 & 22 & 25.97 \\
\hline 22 & $8.0-11.0$ & - 33 & $5^{2}$ & +61 & 9 & 10.12 \\
\hline 23 & $11.0-12.0$ & - $3^{6}$ & 10.83 & $+6 I$ & 30 & 51.54 \\
\hline 24 & $12.0-12.0$ & - 36 & 35.91 & +59 & 57 & 57.62 \\
\hline 25 & $10.8-12.0$ & 041 & 13.16 & +59 & 49 & 11.38 \\
\hline 26 & $10.0-11.0$ & $\circ 43$ & 24.63 & +60 & 27 & 44.97 \\
\hline 27 & $11.0-11.8$ & 043 & 24.66 & +60 & 27 & 46.14 \\
\hline 28 & $9.0-12.5$ & 043 & 44.09 & +60 & 12 & 51.41 \\
\hline 29 & $8.0-12.0$ & 043 & 44.15 & +60 & 12 & 45.11 \\
\hline 30 & $12.5-12.5$ & o 48 & 44.53 & +60 & 35 & $5^{6.92}$ \\
\hline $3 \mathbf{I}$ & $11.2-12.8$ & 049 & 56.11 & +60 & 6 & 17.56 \\
\hline 32 & $11.0-11.0$ & - 53 & 13.85 & +59 & 10 & $34 \cdot 36$ \\
\hline 33 & $10.1-10.3$ & 057 & 6.00 & +59 & 59 & 32.16 \\
\hline 34 & $9.0-11.0$ & 057 & 22.75 & $+5^{8}$ & 57 & 0.71 \\
\hline
\end{tabular}

rinvenute nelle lastre fotografiche pubblicate nel $1^{\circ}$ Volume del Catalogo fotografico stellare corrispondente alla zona Vaticana.

Di P. Angelo Rodriguez, O. S. A., Direttore della Specola Vaticana. 